\title{
Características de um grupo de usuários do Programa Saúde da Família na cidade de Campo Bom (RS), Brasil em 2006
}

\author{
Characteristics of Family H ealth Program users sample \\ in Campo Bom (RS), Brazil during the year of 2006
}

Bárbara N iegia Garcia de Goulart ${ }^{1}$

Andressa Regina Algayer ${ }^{1}$

${ }^{1}$ Instituto de Ciências da Saúde, Feevale. Rodovia RS 239 2755, Bairro Vila Nova. 93352-000 Novo Hamburgo RS. bgoulart@via-rs.net
Abstract This study aims to describe the characteristics of Family Health Program (FHP) users sample in a Basic Health U nit (BHU) in Campo Bom, southern of Brazil. Based on data collected from the handbook of the first 200 patients attended in a specific BHU in January 2006, we analyzed sex, age, average number of medical and nursing consultations in the last twelve months and the reasons for requiring a $\mathrm{BHU}$ attendance. In this sample, $68 \%$ werefemale. Children represented $30.5 \%$ of the sampleand $35.5 \%$ of the patients self-declared as married. In addition to that, 59\% had not finished basic school. Almost half ( $42 \%$ ) of the citizens presented some chronic disease as a complaint to go to a BUH and $17 \%$ were looking for preventive health care. In $2005,17 \%$ of the sample had, at least, one nursing consulting and $46.5 \%$ of these had from one to four medical consultations. Chronicle diseases and its symptoms or complications represented a significant percentage of the sample and might be associated to the demands for BHU health assistance. Data collected shows the necessity of more investments to prevent complications and/or incapability. Based on a deep understanding of BHU patients' profile, family health teams can improve effectiveness of their health promotion actions focusing on community demands.

Key words Family Health Program, Epidemiological studies, Epidemi ology, H ealth services, SUS, Unified $\mathrm{H}$ ealth System
Resumo 0 objetivo deste estudo é conhecer as características de uma amostra da população atendida por uma EquipedeSaúde da Família que buscou atendimento em uma unidade de saúde (UBS) do município de Campo Bom (RS) em 2006. Foram selecionados os primeiros 200 pacientes que consultaram a UBS pesquisada no mês de janeiro de 2006 ejá consultavam no serviço.Características pessoais eligadas à busca por atendimento nos últimos doze meses foram verificadas. Dos sujeitos da amostra, $68 \%$ eram do sexo feminino. As crianças representaram $30,5 \%$ e $59 \%$ dos entrevistados apresentavam ensino fundamental incompleto. A doença crônica como motivo para procura pela UBS ocorreu em $42 \%$ dos sujeitos e $17 \%$ procuraram o serviço para prevenção primária. As doenças crônicas representaram um percentual alto da amostra e provavelmente estão associadas à busca de atendimento clínico na UBS em virtude dos sintomas e complicações decorrentes destas. Este dado demonstra a necessidade de investimentos na prevenção de incapacidades e/ou complicações e prevenção da instalação de doenças crônicas preveníveis. A partir do conhecimento mais detal hado do perfil dos usuários do serviço de saúdeestudado, a equipede saúde pode promover uma atuação mais efetiva.

Palavras-chave Programa Saúde da Família, Estudos epidemiológicos, Epidemiologia, Serviços de saúde, Sistema Ú nico de Saúde 
Introdução

A saúde coletiva éum assunto imensamentediscutido em todo o país. Desde a criação do Sistema Ú nico deSaúde(SUS) e baseado em seus princípios, foram empreendidos diversos esforços pelos governos federal, estaduais e municipais, população e profissionais de saúde para que seus preceitos sejam devidamente respeitados e implementados $^{1-5}$. Muitas propostas e controvérsias sobre as formas de implementação efetiva de políticas intersetoriais que garantam acesso universal, integral e diminuição das desigualdades sociais vêm sendo apresentadas na literatura nacional e encontros ligados ao setor saúde ${ }^{2,5-9}$.

Além disso, a saúde é um conceito complexo. Podemos afirmar que não se trata apenas da ausência de doença e, sim, de um conjunto de aspectos sociais e culturais da vida de cada indivíduo 2,10,11. Portanto, promover a saúde não está somente relacionado à cura das doenças.

Cabe aos profissionais da saúde debruçar-se, nosníveis locais, conhecendo a realidade dosusuários, para inserir-se na comunidade de forma efetiva, contribuindo com a promoção de mudanças que colaborem para a melhoria da qualidade de vida da população e, por consequência, melhora das condições para manutenção da saúde e prevenção de agravos ${ }^{11-15}$.

As propostas contemporâneas de saúde coletiva devem privilegiar as práticas preventivas e a adequação das propostas de saúde às demandas regionais $5^{2,7,12,15,16}$. N este contexto, o Programa Saúde da Família (PSF) está intimamente ligado aos pressupostos do Sistema Ú nico de Saúde; talvez suas propostas sejam a tradução mais completa desses princípios ${ }^{12,16,17}$.

O Programa Saúde da Família está pautado, dentre outras diretrizes, no trabalho em equipe multiprofissional e na participação e controle social. Entende-se que ambas as diretrizes estão relacionadas na medida em que decorrem e expressam as relações entrea população de referência eo serviço ea equipe de trabalho, bem como, em um plano ainda mais microscópico, as relações entretrabalhadores eusuários ${ }^{9,15,18}$. Ambas as propostas, de participação social e do trabalho em equipe, supõem um processo de democratização das instituiçõeses,18.

A inclusão da família como foco de atenção básica de saúde pode ser ressaltada como um dos avanços, como contribuição do PSF para modificar o modelo biomédico de cuidado em saúde ${ }^{8,15,16}$. Os autores destacam que essa estratégia ultrapassa o cuidado individualizado, focado na doença; elege-se aquele que contextualiza a saúde, produzida em um espaço físico, social, relacional, resgatando as múltiplas dimensões da saúde. Ressalta-se que essa inclusão não decorreu deentendimento e convicção pactuadas; viu-se que a família chega à atenção primária de saúde impulsionada por diferentes e conflitantes representações e motivações, daí as facilidades de se produzirem contradições.

Então, é de fundamental importância que a equipe de saúde conheça o perfil da comunidade na qual está inserida, suas demandas e peculiaridades, a fim de que as propostas de preservação, manutenção e restauração da saúde sejam mais diretas e eficientes.

0 objetivo deste estudo é conhecer as características da população atendida por uma Equipe de Saúde da Família que buscaram atendimento no período do estudo em uma Unidade de Saúde do município de Campo Bom (RS), a fim de contribuir com a melhora da efetividade das ações da equipe de saúde da família no sentido de diminuir a demanda por atendimento clínico para prevenção de sequelas ou consequências de doenças ou agravos preveníveis.

\section{M étodo}

Estudo transversal realizado a partir do prontuário dos sujeitos que residem na cidade de Campo Bom (RS) e que frequentaram a Unidade Básica de Saúde Yedda Emília Fauth Blos (popularmente conhecida como UBS 25 de Julho).

Fizeram parte do estudo os moradores dos bairros 25 de Julho e M ônaco que estão vinculados a UBS Yedda Emília Fauth Blos por meio de cadastramento, realizado pelas agentes comunitárias de saúde (ACS). Fizeram efetivamente parte da amostra estudada os primeiros 200 usuários que frequentaram a Unidade Básica de Saúde a partir de janeiro de 2006. Os dados foram coletados nos prontuários dos pacientes.

Foram incluídos todos os indivíduos que receberam atendimento de profissionais que constituem a Equipe de Saúde da Família: médico, enfermeira e técnicos de enfermagem.

Foram excluídos os atendimentos feitos exclusivamente por recepcionista, serviços gerais eagentes de saúde.

Este projeto foi analisado e aprovado pelo Comitê de Ética em Pesquisa do Centro Universitário Feevale ( processo n ${ }^{\circ}$ 4.06.03.05.175), conformedispositivos contidos na resolução CNS 196/96. A autorização para uso dos dados na pesquisa foi solicitada à Secretaria M unicipal da Saúde de Campo Bom e à coordenação da UBS Yedda Emília 
Fauth Blos. Também foi realizado um termo de compromisso para utilização de dados, assinado pelos pesquisadores.

No período delimitado da coleta de dados, foram analisados os prontuários dos usuários que realizaram consultas(s) médica (s) ou de enfermagem na Unidade de Saúde pesquisada. Foram levantados dados sobre local de residência, sexo, idade, grau de escolaridade, situação familiar (matrimonial), profissão, motivo de procura por atendimento e histórico de doença crônica, além do número de consultas de enfermagem, médicas e acolhimentos realizados por técnicos de enfermagem no período de 10 dejaneiro a 31 de dezembro de 2005.

Em relação ao local de residência, os sujeitos poderiam ser procedentes do bairro 25 de Julho ou do bairro M ônaco; a situação familiar (matrimonial) foi classificada em casado ou concubinato, solteiro, viúvo, separado ou divorciado e não se aplica no caso de crianças; o histórico de doença crônica classificado em existenteou inexistente. Em relação ao motivo de procura por atendimento, ou seja, o fato que levou o indivíduo a procurar a Unidade Básica deSaúde, podemos classificar em atendimento preventivo (consultas de pré natal, coleta decitopatológico e puericultura), dor, retorno (mostrar exames), alergias, distúrbios gastrintestinais (diarréia e ou vômito), solicitação de exames, problemas do sistema tegumentar (lesões de pele, mucosa oral e genital), otites, amigdalites, outros (broncoespasmo, febre, ansiedade, tosse, tonturas, gripe, aumento de peso, amenorréia, trauma, preenchimento delaudos, hematúria, cansaço, diabete mellitus descompensado, conjuntivite, insônia, anemia) e solicitação de receitas e encaminhamentos.

Todas as análises estatísticas foram feitas a partir do programa Epilnfo, versão 3.3.2. Foram analisados dados ligados ao bairro de moradia, idade, sexo, histórico de doença crônica, situação familiar /matrimonial, tipo de atendimento, escolaridade, motivo de procura por atendimento, número mé dio de consultas no intervalo detempo pesquisado.

\section{Resultados ediscussão}

A população total de Campo Bom no período estudado era estimada em 58.558 habitantes, conforme dados do DATASU ${ }^{19}$. Deste total, $12 \%$ estavam cobertos pelo PSF em 2006 e a média de consultas médicas em especialidades básicas foi de 0,58 por habitante no período ${ }^{20}$.

Dentre os 200 sujeitos pesquisados, 158 (79\%) residiam no bairro 25 de Julho e 136 (68\%) eram do sexo feminino. 0 fato de os moradores que mais frequentaram a Unidade de Saúde serem do bairro 25 de Julho provavelmente está associado à proximidade maior deste em relação à UBS, além desta população representar $87 \%$ do total de indivíduos cadastrados no PSF daquela região $0^{1,2,8,12}$.

Concordando com dados publicados por outros autores, foram as mulheres quem mais procuraram o serviço de saúde ${ }^{21,23}$. Observamos que o número de mulheres cadastradas pela UBS também é proporcionalmente maior em relação aos

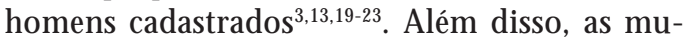
Iheres estudadas atuam principalmente nas tarefas domésticas e criação dos filhos.

Da amostra de usuários atendidos na UBS no período, 61 (30,5\%) eram crianças de 0 a 10 anose $46(23 \%)$, adultos com idades entre 40 e 60 anos. Estes dados contrariam os achados de outros estudos ${ }^{23,24}$, no qual os autores observaram que os idosos eram os principais usuários de serviços de saúde, atribuindo este fenômeno à maior prevalência de doenças e outros agravos nesta população. A população da cidade no período do estudo tinha em torno de $10 \%$ de crianças com idades entre 0 e 4 anos e $27,9 \%$ da população total do município tinha 40 anos ou mais, conforme dados disponíveis no DATASU ${ }^{19}$.

Comparando os dados populacionais da cidade com as características dos usuários da UBS no período pesquisado, constatamos que, em média, um percentual menor da população atendida pelo PSF chegou ao atendimento na UBS. Isso pode demonstrar a efetiva contribuição das ações promovidas pelas equipes de saúde da família no sentido de promover a saúde e prevenir agravos ou doenças, evitando a necessidade de atendimento naUBS.

O levantamento dos prontuários apontou que 71 (35,5\%) dos usuários pesquisados são casados, porém não foram encontrados dados bibliográficos relacionados a esta variável. Ressaltamos que tal dado é fundamental para que se conheça mais profundamente a situação de vida dos usuários do serviço de saúde, constituindo informação essencial para a construção de programas de planejamento familiar ede doenças sexualmentetransmissíveis direcionados a esta população que possui companheiro fixo, além de outras questões possivelmente ligadas à situação familiar matrimonial.

Quanto à escolaridade, 118 (59\%) sujeitos referiram ensino fundamental incompleto. Observa-seque o tempo de estudo destes usuários é próximo ao que se encontrou na literatura. Campo Bom é uma cidade basicamente urbana e industrial. 0 índice deanalfabetismo éde 5,1\% emais de 
5\% da população têm menos de um ano de estudo. A média do grau de instrução da população é de 5,1 anos de estudo e a cobertura de saneamento básico é quase total ${ }^{20}$.

Este dado pode trazer subsídios sobre as características necessárias ao contato, relacionamento e comunicação com estes sujeitos, buscan do a utilização de estratégias que favoreçam sua compreensão das proposições e indicações ligadas ao tratamento de doenças e agravos, bem como ações de promoção e manutenção da saúde.

Constatou-se que 84 (42\%) dos sujeitos estudados têm algum tipo de doença crônica, fato já descrito em 199723, quando outro estudo no mesmo município demonstrou que os usuários adultos que mais frequentam os serviços de saúde, em geral, têm hipertensão ou algum problema crônico. A análise multivariada dos autores também confirmou que, quanto pior a condição socioeconômica do paciente, maior a utilização do sistema público de saúde.

Dentre os 153 usuários adultos pesquisados, $34(22,2 \%)$ eram industriários, $32(20,9 \%)$ eram estudantes, $26(17 \%)$ declararam-se aposentados ou pensionistas e $42(27,4 \%)$ apresentaram outra atividade profissional. A atividade laboral mais comum esteve relacionada ao trabalho na indústria. Esta característica pode ser associada ao fato de Campo Bom ser uma cidade industrializada e ao fato de que, para este tipo de atuação, não há exigência na região em relação à escolaridade ${ }^{20,21}$.

Em 19 (12,4\%) dos prontuários pesquisados, o campo referente à ocupação (ou profissão) não estava preenchido. A partir disso, destacamos a necessidade de que os prontuários e outros documentos ligados à situação de cada paciente sejam preenchidos naíntegra, pois podem constituir importante subsídio para decisões sobre os encaminhamentos em relação à atenção ao paciente na sua integralidade.

Tabela 1. Proporção e intervalo de confiança (95\%) de consultas de enfermagem anuais para a população pesquisada.

\begin{tabular}{lrrr}
\hline $\begin{array}{l}\text { No de consultas } \\
\text { de enfermagem }\end{array}$ & $\mathrm{N}$ & $\%$ & $\%$ cumulativa \\
\hline 0 & 111 & 55,5 & - \\
1 & 34 & 17,0 & 72,5 \\
2 & 28 & 14,0 & 86,5 \\
3 ou + & 27 & 13,5 & 100 \\
Total & 200 & 100 & - \\
\hline
\end{tabular}

É necessário que a equipe de saúde conheça os usuários que frequentam a unidade de saúde, incluindo sua ocupação, pois é importante que se procure relacionar os motivos de consultas com as atividades laborais. A partir de tais correlações, é possível estabelecer condutas preventivas, tanto para evitar a piora da evolução da doença, quanto para evitar que o dano leve à impossibilidade de continuidade na atividade laboral costumeira do paciente.

Em relação ao número de consultas de enfermagem, no período de um ano, 111 sujeitos $(55,5 \%)$ não realizaram nenhuma, conforme descrito na Tabela 1.

No período pesquisado, $46,5 \%$ (93) dos sujeitos da amostra realizaram de 1 a 4 consultas médicas, conforme descrito na Tabela 2.

No ano de 2005, 63,5\% (127) dos usuários não realizaram nenhum acolhimento (atendimento prestado pelos técnicos de enfermagem) e 18,5\% (37) realizaram apenas um acolhimento.

Em outro estudo com objetivo de levantar características relacionadas à ida na UBS por ido$\operatorname{sos}^{24,25}$, os autoresobservaram que a média de consultas médicas da população idosa em um período de doze meses é de três para $44,3 \%$ dos idosos pesquisados, 27,8\% não tiveram nenhuma consulta, $14 \%$ dos idosos tiveram duas consultas e 13,9\% tiveram uma consulta neste mesmo período.

Em nossa experiência, a população estudada tem uma média de consultas médicas em um período de doze meses bastante semel hante aos achados encontrados na pesquisa citada anteriormente. Entretanto, caberessaltar, esteestudo conta com população com idade variada eatendida pelo Programa Saúde da Família. Os dados são semelhantes àqueles trazidos nos indicadores de saúde do município no ano de 2006 (0,58 consulta médica em especialidade básica por habitante) ${ }^{19}$.

Tabela 2. Proporção e intervalo de confiança (95\%) de consultas médicas anuais para a população pesquisada.

\begin{tabular}{lrrr}
\hline $\begin{array}{l}\text { No de consultas } \\
\text { médicas }\end{array}$ & N & $\%$ & $\%$ cumulativa \\
\hline 0 & 42 & 21,0 & - \\
$1-4$ & 93 & 46,5 & 67,5 \\
$5-8$ & 44 & 22,0 & 89,5 \\
9 ou + & 21 & 10,5 & 100,0 \\
Total & 200 & 100,0 & - \\
\hline
\end{tabular}


Os dados encontrados em nosso estudo estão de acordo com publicação de Rebelo ${ }^{25}$, que apontou que $70 \%$ da população procuram atendimento médico pelo menos uma vez à cada doze meses. Entretanto, não se confirma em nosso estudo a afirmação de que nas idades mais avançadas há uma tendência de comparecer mais ao médico, pois nestas faixas etárias são mais frequentes os problemas de saúde, visto que a distribuição das idades na população estudada foi semelhante nas diversas faixas etárias.

Em relação aos motivos de procura por atendimento, dentre os 200 sujeitos pesquisados, $42 \%$ apresentam algum tipo de doença crônica como motivo para procura pela UBS e 34 (17\%) procuraram o serviço para prevenção primária.

Os achados acima corroboram estudo prévio que apontou como principais motivos de consulta na U BS aqueles ligados à hipertensão, doenças osteomusculares e doenças sazonais (IRA e diarréia) ${ }^{21}$. O utro estudo, publicado em $1997^{23}$, detectou que $47 \%$ da demanda da UBS seriam motivados por doenças crônicas e apenas 18\% decorrem de demandas ligadas à prevenção. 0 s autores destacaram que os serviços de saúde pesquisados tinham atividades predominantemente curativas ou paliativas, considerando a demanda observada. Em nosso estudo, os dados mostram-se semelhantes e estão de acordo com os indicadores da cidade de Campo Bom para o ano de $2006^{19}$.

0 objetivo geral da Estratégia da Saúde da Família é reorientar o modelo assistencial de saúde, imprimindo uma nova dinâmica de atuação e de relacionamento entre os serviços de saúde e a população ${ }^{2,12,15,18}$. Para atingi-lo, épreciso quea equipe conheça a realidade da população atendida, das organizações familiares e comunitárias, desenvolvendo um processo de planejamento a partir desta realidade, para executar ações compatíveis com as necessidades ${ }^{4,9,10,14}$.

Quando relacionamos os dados deste estudo com os dados do município pesquisado para o ano de $2006^{19}$, constatamos que não parece haver diferença estatisticamentesignificativa entre as demandas de busca por atenção em unidade básica de saúde em região abrangida ou não pelo PSF. Além disso, a dinâmica do serviço de saúde ainda mostra-se pautada pela atenção à saúde baseada na livre demanda do sujeito. Ou seja, a pessoa busca o serviço de saúde em virtude de necessidade de atendimento e este é um importante contraponto à proposta do PSF, que tem como objetivo, entre outros, a reorganização do serviço de saúde e meIhora da resolutividade. Os indicadores referentes a número de visitas domiciliares por família em
2006 apontam para 0,17 visita/mês por família abrangida pelo PSF naquele ano ${ }^{19}$.

Este trabal ho busca retomar a importância dos estudos de (re)conhecimento de perfis populacionais, seja de uma comunidade, seja da população atendida em um serviço ligado ao setor saúde. Reforçamos a necessidade de que as práticas na esfera da saúde pública sejam embasadas por forte evidência clínico-epidemiológica e venham ao encontro dos pressupostos do SUS e da estratégia de saúde da família, estando acima de qualquer entidade ou classe profissional ${ }^{14,22}$.

\section{Consideraçõesfinais}

Constatamos que os atendimentos curativos (prevenção secundária, terciária) são proporcionalmente mais numerosos que os atendimentos ligados à prevenção primária.

O levantamento eanálise das características dos pacientes que buscam atendimento na UBS devem ser considerados quando da proposição de ações para a comuni dadee tais iniciativas devem ser uma constante na avaliação emonitoramento das ações e serviços de saúde.

É preciso considerar que, além de influenciada pelo perfil de necessidades de cada grupo populacional, as características dos usuários dos serviços de saúde e suas demandas estão condicionadas por inúmeros outros fatores (internos e externos), relacionadas tanto à oferta dos serviços quanto às preferências e possibilidades dos usuários.

É necessário ampliar ainda mais as formas de atenção à saúde, promovendo ações de prevenção e educação para a promoção da saúde. Tais avanços devem contribuir com o fortalecimento da implementação do SUS, conformepreconizado em suas diretrizes, além de colaborar para a melhora das condições de acesso aos serviços de saúde, bem como sua resolutividade, trazendo benefícios diretos para os usuários.

\section{Colaboradores}

BN G Goulart e AR Algayer participaram da concepção do estudo, coleta e análise dos dados, redação e revisão do texto. 
Referências

1. Ceccim RB, Armani TB, Rocha CF. O que dizem a Legislação e o Controle Social em Saúde sobre a Formação de Recursos Humanos e o Papel dos Gestores Públicos, no Brasil. Cien Saude Colet 2002; 7 (2):373-383.

2. Reis CCL, Hortale VA. Programa Saúde da Família: supervisão ou "convisão"? Estudo de caso em município de médio porte. Cad Saude Publica 2004; 20(2):492501.

3. Franco ALS, Bastos ACS, Alves VS. A relação médico-paciente no Programa Saúde da Família: um estudo em três municípios do Estado da Bahia, Brasil. Cad Saude Publica 2005; 21 (1):246-255.

4. Silva IZQJ, Trad LAB. O Trabalho em Equipe no PSF: investigando a articulação técnica e a interação entre os profissionais. Interface (Botucatu) 2005; 9(16):25-38.

5. Sakai M H, Nunes EFPA, M artins VL, Almeida MJ, Baduy RS. Recursos Humanos em Saúde. In: Andrade SM, Soares DA, Cordoni-Junior L, organizadores. Bases da Saúde Coletiva. Londrina: UEL; 2001. p.111-124.

6. Salsano FM. Saúde Pública no Primeiro e Terceiro Mundos: desafios e perspectivas. Cien Saude Colet 2002; 7(1):7-16.

7. Goulart BNG. A Fonoaudiologia e suas Inserções no Sistema Ú nico de Saúde: análise prospectiva. Rev Fonoaudiol Brasil 2003; 2(4):29-34.

8. Ribeiro EM. As Várias Abordagens da Família no Ce nário do Programa/Estratégia de Saúde da Família (PSF). Rev Latino-am enfermagem 2004; 12 (4):658-664.

9. Crevelim MA. Participação da Comunidade na Equipe de Saúde da Família: é possível estabelecer um projeto comum entre trabalhadores e usuários? Cien Saude Colet 2005; 10(2):323-331.

10. Aerts D, Alves GG, La Salvia MW, Abegg C. Promoção de saúde: a convergência entre as propostas da vigilância da saúde e da escola cidadã. Cad Saude Publica 2004; 20(4):1020-1028.

11. Coelho MTAD, Almeida Filho N. Concepções populares de normalidade e saúde mental no litoral norte da Bahia, Brasil. Cad Saude Publica 2005; 21(6):17261736.

12. Senna M CM. Equidade e Política de Saúde: algumas reflexões sobre o Programa Saúde da Família. Cad Saude Publica 2002; 18(Supl.):203-211.

13. Goldbaum M, Gianin RJ, Novaes HMD, César CLG. Utilização de Serviços de Saúde em Áreas Cobertas pelo Programa Saúde da Família (Qualis) no M unicípio de São Paulo. Rev. Saude Publica 2005; 39(1):90-99.

14. Goulart BNG, Chiari BM. Construção e Aplicação de Indicadores de Saúde na Perspectiva Fonoaudiológica - contribuições para a reflexão. Rev Soc Bras Fonoaudiol 2006; 11(3):194-204.

15. Bertussi DC, Oliveira MSM, Lima JVC. A Unidade Básica no Contexto do Sistema de Saúde. In: Andrade SM, Soares DA, Cordoni-Junior L, organizadores. Bases da Saúde Coletiva. Londrina: UEL; 2001. p.133-144.
16. Ribeiro EM, Chomatas VER, N eto MC. Integralidade e Redes de Atenção Básica e Saúde Familiar: a experiência do município de Curitiba-PR. Projeto de Desenvolvimento de Sistemas e Serviços de Saúde. Brasília: OPAS/ Ministério da Saúde; 2004. p. 37-61.

17. Cohen SC, Cynamon SE, Kligerman DC, Amaral LCP, M esquita L, Ribeiro LP. Rede Brasileira de Habitação Saudável como Estratégia Sinérgica da Saúde da Família no Brasil. Projeto de Desenvolvimento de Sistemas e Serviços de Saúde. Brasília: OPAS/M inistério da Saúde; 2004. p.103-116.

18. Carnelosso ML, Barbosa MA, Souza ALL, Monego ET, Carvalho MM. Enfermidades não-transmissíveis na Atenção Básica: novo desafio para o PSF. Projeto de Desenvolvimento de Sistemas e Serviços de Saúde. Brasília: OPAS/M inistério da Saúde; 2004. p. 117-126.

19. Brasil. M inistério da Saúde. Departamento de Informática do SUS. Cadernos do Pacto de Atenção Básica 2006 - Campo Bom, Rio Grande do Sul. [acessado 2008 jan 05]. Disponível em: http://tabnet.datasus.gov. br/ tabdata/pacto2006/RS/RS_Campo_Bom_Pacto2006 Series.xls

20. González M M. Análise de Situação e Proposta Estratégica para 2004. Campo Bom: Secretaria Municipal de Saúde; 2003.

21. Vargas MD, Seghetto R. Análise de Situação e Proposta Estratégica da Equipe de Saúde da Família da U nidade Básica de Saúde 25 de Julho. Campo Bom: Secretaria Municipal de Saúde; 2003.

22. Vaughan JP, Morrow RH. Epidemiologia para Municípios. M anual para Gerenciamento dos Distritos Sanitários. 3ạ ed. São Paulo: Hucitec; 2002.

23. Costa JD, Facchini LM. Utilização de serviços ambulatoriais em Pelotas: onde a população consulta e com que frequência. Rev. Saude Publica 1997; $31(4): 360-369$.

24. Costa-Lima M F. Epidemiologia do envelhecimento no Brasil. In: Rouquayrol MZ, Almeida Filho N. Epidemiologia \& Saúde. 6a ed. Rio de Janeiro: MEDSI; 2003. p. 499-514.

25. Rebelo PAP. Qualidade em Saúde: modelo teórico, realidade, utopia e tendência. Rio de Janeiro: Qualitymark; 1995.

Artigo apresentado em 04/08/2007

Versão final apresentada em 14/12/2007 